Pacific

Journal of

Mathematics

\title{
GROUP-GRADED RINGS AND FINITE BLOCK THEORY
}

YUN FAN AND BURKHARD KÜLShammeR 


\title{
GROUP-GRADED RINGS AND FINITE BLOCK THEORY
}

\author{
YUN FAN AND BURKHARD KÜLSHAMMER
}

\begin{abstract}
Affirmative answers to two questions of Dade are given: 1 . If the 1-component $R_{1}$ of a ring $R$ graded by a finite group contains only finitely many central idempotents then so does $R$. 2. If $R$ is a ring fully graded by a finite group $G$ and if $S$ is a $G$-invariant unitary subring of $R$ then, for every block idempotent $a$ of $R$, the block idempotents $b$ of $S$ such that $a b \neq 0$ form a single $G$-orbit.
\end{abstract}

\section{Notation and terminology.}

All rings in this paper will be associative with identity element. For a ring $R$, we denote by $\mathrm{Z}(R)$ its center, by $\mathrm{J}(R)$ its Jacobson radical, and by $\mathrm{U}(R)$ its group of units. Note that an element $r \in R$ is contained in $\mathrm{U}(R)$ if and only if its residue class $r+\mathrm{J}(R)$ is contained in $\mathrm{U}(R / \mathrm{J}(R))$.

A homomorphism of rings $\phi: R \rightarrow S$ is not required to satisfy $\phi\left(1_{R}\right)=1_{S}$; if it does then we say that $\phi$ is unitary. Similarly, a subring $S$ of a ring $R$ is not required to satisfy $1_{S}=1_{R}$; if it does then we call $S$ a unitary subring of $R$.

An element $e \in R$ such that $e^{2}=e$ is called an idempotent. It is wellknown that 0 is the only idempotent of $R$ which is contained in $\mathrm{J}(R)$. Two idempotents $e, f \in R$ are called orthogonal if ef $=0=f e$. A nonzero idempotent $e \in R$ is called primitive in $R$ if it is impossible to write $e=f+g$ with nonzero orthogonal idempotents $f, g \in R$. A block idempotent of $R$ is an idempotent in $\mathrm{Z}(R)$ which is primitive in $\mathrm{Z}(R)$. We denote the set of block idempotents of $R$ by $\operatorname{Bl}(R)$.

Following [1], we say that $R$ has finite block theory if $\mathrm{Z}(R)$ contains only finitely many idempotents. By [1, Proposition 1.4], this is equivalent to saying that $1_{R}$ can be written as a finite sum $1_{R}=b_{1}+\cdots+b_{m}$ of block idempotents $b_{1}, \ldots, b_{m} \in \mathrm{Bl}(R)$. In this case we even have $\mathrm{Bl}(R)=$ $\left\{b_{1}, \ldots, b_{m}\right\}$. We note that $|\mathrm{Bl}(R)|<\infty$ alone does not guarantee that $R$ has finite block theory.

Throughout this paper, we will work with a fixed finite group $G$. A $G$-ring is a ring $R$, together with an action of $G$ on $R$ via ring automorphisms. We denote by ${ }^{x} r$ the image of an element $r \in R$ under an element $x \in G$, and by $R^{G}=\left\{r \in R:{ }^{x} r=r\right.$ for $\left.x \in G\right\}$ the fixed point subring of $R$ under $G$. It is shown in [1, Theorem 2.2] that $R$ has finite block theory if $R^{G}$ has. 
A $G$-graded ring is a ring $R$, together with a fixed decomposition $R=$ $\bigoplus_{x \in G} R_{x}$ into additive subgroups $R_{x}$ (the $x$-components of $R$ ) such that $R_{x} R_{y} \subseteq R_{x y}$ for $x, y \in G$; here $R_{x} R_{y}$ denotes the additive subgroup of $R$ consisting of all finite sums of elements $r_{x} s_{y}$ with $r_{x} \in R_{x}$ and $s_{y} \in R_{y}$. The 1-component $R_{1}$ of $R$ is always a unitary subring of $R$, and $\mathrm{J}\left(R_{1}\right)=R_{1} \cap \mathrm{J}(R)$ by $[\mathbf{2}$, Corollary $2(\mathrm{c})]$.

A graded subring of $R$ is a subring $S$ of $R$ such that $S=\bigoplus_{x \in G}\left(S \cap R_{x}\right)$. In this case $S$ itself becomes a $G$-graded ring with $x$-component $S_{x}=S \cap R_{x}$ for $x \in G$. Similarly, a graded ideal of $R$ is an ideal $I$ of $R$ such that $I=\bigoplus_{x \in G}\left(I \cap R_{x}\right)$. In this case, $I_{x}=I \cap R_{x}$ is called the $x$-component of $I$ for $x \in G$. Moreover, $R / I$ becomes a $G$-graded ring with $x$-component $(R / I)_{x}=\left(R_{x}+I / I\right) \cong R_{x} / I_{x}$ for $x \in G$.

If $R^{\prime}=\bigoplus_{x \in G} R_{x}^{\prime}$ is another $G$-graded ring then a graded homomorphism from $R$ to $R^{\prime}$ is a ring homomorphism $\phi: R \rightarrow R^{\prime}$ such that $\phi\left(R_{x}\right) \subseteq R_{x}^{\prime}$ for $x \in G$. Then the kernel $\operatorname{Ker}(\phi)$ is a graded ideal of $R$ while the image $\phi(R)$ is a graded subring of $R^{\prime}$. Conversely, for a graded subring $S$ of $R$ and a graded ideal $I$ of $R$, the canonical maps $S \rightarrow R$ and $R \rightarrow R / I$ are graded homomorphisms.

If $R=\bigoplus_{x \in G} R_{x}$ is a $G$-graded ring then the centralizer

$$
C=\mathrm{C}_{R}\left(R_{1}\right)=\left\{c \in R: c r_{1}=r_{1} c \text { for } r_{1} \in R_{1}\right\}
$$

of $R_{1}$ in $R$ is a $G$-graded unitary subring of $R$. The 1 -component $C_{1}=\mathrm{Z}\left(R_{1}\right)$ of $C$ is contained in $\mathrm{Z}(C)$, and so is $\mathrm{Z}(R)$. Thus, if $C$ has finite block theory then so has $R$, and $|\mathrm{Bl}(R)| \leq|\mathrm{Bl}(C)|$ in this case.

A $G$-graded ring $R=\bigoplus_{x \in G} R_{x}$ is called fully graded (resp. a crossed product) if $R_{x} R_{x^{-1}}=R_{1}$ for $x \in G$ (resp. if $R \neq 0$ and $R_{x} \cap \mathrm{U}(R) \neq \emptyset$ for $x \in G)$. Of course, every crossed product is fully graded. If $R=\bigoplus_{x \in G} R_{x}$ is fully graded then there is a canonical action of $G$ on $C=\mathrm{C}_{R}\left(R_{1}\right)$ via ring automorphisms (cf. [1, Lemma 5.1]), and $C$ becomes a $G$-ring with fixed point subring $C^{G}=\mathrm{Z}(R)$. Moreover, the $G$-action on $C$ is compatible with the $G$-grading of $C$, in the sense that ${ }^{x} C_{y}=C_{x y x^{-1}}$ for $x, y \in G$.

A subring $S$ of a fully $G$-graded $\operatorname{ring} R=\bigoplus_{x \in G} R_{x}$ is called $G$-invariant if $R_{x} S R_{x^{-1}}=S$ for $x \in G$. In this case $\mathrm{Z}(S)$ is a $G$-subring of $C=\mathrm{C}_{R}\left(R_{1}\right)$, by $[\mathbf{1}$, Proposition 8.3$]$.

\section{The main results.}

Our first main result gives a positive answer to Question 10.1 in [1].

Theorem 1. Let $G$ be a finite group, and let $R=\bigoplus_{x \in G} R_{x}$ be a G-graded ring such that the 1-component $R_{1}$ of $R$ has finite block theory. Then $R$ has finite block theory, and $|\mathrm{Bl}(R)| \leq|G| \cdot\left|\mathrm{Bl}\left(R_{1}\right)\right|$.

Theorem 1 will follow from the next result which gives a more precise description of the situation in a special case. 
Theorem 2. Let $G$ be a finite group, and let $R=\bigoplus_{x \in G} R_{x}$ be a $G$-graded ring such that $R_{1}$ has finite block theory and $R_{1} \subseteq \mathrm{Z}(R)$. If $E$ is a set of pairwise orthogonal nonzero idempotents in $R$ then $|E| \leq|G| \cdot\left|\operatorname{Bl}\left(R_{1}\right)\right|$.

Our next main result gives a positive answer to Question 10.3 in [1].

Theorem 3. Let $G$ be a finite group, let $S$ be a $G$-invariant unitary subring of a fully G-graded ring $R=\bigoplus_{x \in G} R_{x}$, and let a be a block idempotent of $R$. Then the block idempotents $b$ of $S$ such that $a b \neq 0$ form a single $G$-orbit $B$, and $a \sum_{b \in B} b=a$.

The proof of Theorem 3 will be a consequence of results in [1], together with the following fact.

Theorem 4. Let $G$ be a finite group, let $R=\bigoplus_{x \in G} R_{x}$ be a $G$-graded ring such that $R_{1} \subseteq \mathrm{Z}(R)$, and let e be a block idempotent in $R$. Then there is a block idempotent $e_{1}$ in $R_{1}$ such that $e e_{1}=e$.

The results above, together with certain facts from [1], lead to the following application to Clifford theory of blocks.

Theorem 5. Let $G$ be a finite group, and let $R=\bigoplus_{x \in G} R_{x}$ be a fully G-graded ring.

(i) For every block idempotent e in $R$, the block idempotents $e_{1}$ in $R_{1}$ such that $e e_{1} \neq 0$ form a single $G$-orbit; in particular, there is at least one, and there are at most finitely many of them.

(ii) Conversely, if $e_{1}$ is a block idempotent in $R_{1}$ then the sum of all $G$ conjugates of $e_{1}$ can be written as a sum of finitely many block idempotents in $R$.

(iii) If $e_{1}$ is a fixed block idempotent of $R_{1}$, and if we set $H=\{y \in G$ : $\left.{ }^{y} e_{1}=e_{1}\right\}$ and $S:=\bigoplus_{y \in H} R_{y}$, then the map $e \mapsto f=e e_{1}$ yields a one-to-one correspondence between block idempotents e in $R$ such that $e e_{1} \neq 0$ and block idempotents $f$ in $S$ such that $f e_{1} \neq 0$.

(iv) If e and $f$ correspond as in (iii) then the rings e $R$ and $f S$ are Morita equivalent.

In Section 3 we will prove some general properties of rings graded by a finite group, and in Section 4 we will consider the special case $R_{1} \subseteq \mathrm{Z}(R)$. Proofs of our main results will be found in Section 5 .

\section{Some general facts.}

We fix a finite group $G$ and a $G$-graded ring $R=\bigoplus_{x \in G} R_{x}$. The following lemma is related to a result in [4].

Lemma 6. Let $I=\bigoplus_{x \in G} I_{x}$ be a $G$-graded ideal of $R$. If there is a subgroup $H$ of $G$ such that $I_{y}=0$ for $y \in H$ then $I^{m}=0$ where $m=|G: H|$. 
Proof. It is clear that $I^{m}=\sum_{x_{1}, \ldots, x_{m} \in G} I_{x_{1}} \cdots I_{x_{m}}$. For any sequence $x_{1}, \ldots, x_{m} \in G$, the $m+1$ cosets $H, x_{1} H, x_{1} x_{2} H, \ldots, x_{1} \ldots x_{m} H$ cannot all be different. Thus two of them coincide, say $x_{1} \ldots x_{s-1} H=x_{1} \ldots x_{s-1} x_{s} \ldots$ $x_{t} H$ where $1 \leq s \leq t \leq m$. Then $x_{s} \ldots x_{t} \in H$, so $I_{x_{1}} \ldots I_{x_{m}} \subseteq R I_{x_{s} \ldots x_{t}} R=$ 0 .

Our next result is an easy consequence of Lemma 6 .

Lemma 7. Let $I=\bigoplus_{x \in G} I_{x}$ be a graded ideal of $R$ such that $I_{1} \subseteq \mathrm{J}\left(R_{1}\right)$. Then $I \subseteq \mathrm{J}(R)$.

Proof. It is easy to see that $R I_{1} R$ is a graded ideal of $R$ with $x$-component $\left(R I_{1} R\right)_{x}=\sum_{y \in G} R_{y} I_{1} R_{y^{-1} x}$ for $x \in G$. Since $R I_{1} R$ is contained in $I$, $I / R I_{1} R$ is a graded ideal of the $G$-graded $\operatorname{ring} R / R I_{1} R$, and its 1 -component is $\left(I / R I_{1} R\right)_{1}=\left(I_{1}+R I_{1} R\right) / R I_{1} R=0$. Hence, by Lemma $6, I / R I_{1} R$ is nilpotent; in particular, we have $I / R I_{1} R \subseteq \mathrm{J}\left(R / R I_{1} R\right)$. On the other hand, [2, Corollary 2(c)] implies that $R I_{1} R \subseteq R \mathrm{~J}\left(R_{1}\right) R \subseteq \mathrm{J}(R)$, so we conclude that $\mathrm{J}\left(R / R I_{1} R\right)=\mathrm{J}(R) / R I_{1} R$. Thus we obtain $I \subseteq \mathrm{J}(R)$.

In the following, we set $G_{R}=\left\{y \in G: R_{y} R_{y^{-1}}=R_{1}\right\}$.

Lemma 8. With notation as above, $G_{R}$ is a subgroup of $G$ such that $R_{x} R_{y}=$ $R_{x y}$ and $R_{y} R_{x}=R_{y x}$ for $x \in G$ and $y \in G_{R}$.

Proof. It is clear that $1 \in G_{R}$. For $x \in G$ and $y \in G_{R}$, we have

$$
R_{y x}=R_{1} R_{y x}=R_{y} R_{y^{-1}} R_{y x} \subseteq R_{y} R_{y^{-1} y x}=R_{y} R_{x} \subseteq R_{y x},
$$

so $R_{y x}=R_{y} R_{x}$. Hence, for $z \in G_{R}$, it follows that

$$
R_{y z} R_{z^{-1} y^{-1}}=R_{y} R_{z} R_{z^{-1} y^{-1}}=R_{y} R_{z z^{-1} y^{-1}}=R_{y} R_{y^{-1}}=R_{1} .
$$

Hence, in particular, we have $y z \in G_{R}$. Since $G$ is finite we conclude that $G_{R}$ is a subgroup of $G$, and

$$
R_{x y}=R_{x y} R_{1}=R_{x y} R_{y^{-1}} R_{y} \subseteq R_{x y y^{-1}} R_{y}=R_{x} R_{y} \subseteq R_{x y}
$$

for $x \in G$ and $y \in G_{R}$.

For a subset $H$ of $G$, we set $R[H]=\bigoplus_{y \in H} R_{y}$. If $H$ is a subgroup of $G$ then $R[H]$ becomes an $H$-graded ring with $y$-component $R[H]_{y}=R_{y}$ for $y \in H$. We denote by $G-H$ the (set-theoretic) complement of $H$ in $G$.

Lemma 9. If $R_{1}$ is a division ring then the $G_{R}$-graded ring $R\left[G_{R}\right]$ is a crossed product, and $R\left[G-G_{R}\right]$ is a nilpotent graded ideal of $R$.

Proof. Let $y \in G_{R}$. Then there are $s \in R_{y}, t \in R_{y^{-1}}$ such that $s t \neq 0$. But st is contained in the division ring $R_{1}$, so st is invertible in both $R_{1}$ and $R$. Thus $s$ has a right inverse in $R$. Note that $0 \neq s t s t$; in particular, we have $0 \neq t s \in R_{1}$. Hence $s$ has a left inverse as well, so $s \in R_{y} \cap \mathrm{U}(R)$. This shows that $R\left[G_{R}\right]$ is a crossed product. 
We claim that $R\left[G-G_{R}\right]$ is an ideal of $R$. By symmetry, it suffices to show that $R\left[G-G_{R}\right]$ is a left ideal. Thus we prove that $R_{x} R_{w} \subseteq R\left[G-G_{R}\right]$ whenever $x \in G$ and $w \in G-G_{R}$. This is trivial in case $x w \in G-G_{R}$. Thus we may assume that $x w \in G_{R}$. Then $x \in G-G_{R}$, so $R_{x} R_{x^{-1}} \neq R_{1}$. But $R_{x} R_{x^{-1}}$ is an ideal in the division ring $R_{1}$, so $R_{x} R_{x^{-1}}=0$. Moreover, since $w^{-1} x^{-1} \in G_{R}$, Lemma 8 implies that $R_{w} R_{w^{-1} x^{-1}}=R_{x^{-1}}$. Thus we conclude that

$$
\begin{aligned}
R_{x} R_{w}=R_{x} R_{w} R_{1} & =R_{x} R_{w} R_{w^{-1} x^{-1}} R_{x w}=R_{x} R_{x^{-1}} R_{x w} \\
& =0 R_{x w}=0 \subseteq R\left[G-G_{R}\right] .
\end{aligned}
$$

This shows that $R\left[G-G_{R}\right]$ is an ideal of $R$. It is clearly graded, and its 1-component is zero. Thus $R\left[G-G_{R}\right]$ is nilpotent by Lemma 6 .

\section{Central 1-components.}

We start by recalling some concepts and facts from commutative algebra. For a commutative ring $A$, we denote by $\operatorname{Spec}(A)$ the spectrum of $A$, i.e., the set of prime ideals of $A$. Then $\operatorname{Spec}(A)$ is a topological space with respect to the Zariski topology; its closed subsets have the form

$$
\mathcal{V}(I)=\{P \in \operatorname{Spec}(A): I \subseteq P\}
$$

where $I$ is a subset of $A$. Thus its open subsets have the form

$$
\mathcal{X}(I)=\{P \in \operatorname{Spec}(A): I \nsubseteq P\}
$$

where $I$ is a subset of $A$. It is well-known that the map

$$
e \longmapsto \mathcal{X}(e)=\{P \in \operatorname{Spec}(A): e \notin P\}
$$

is a bijection between the set of all idempotents $e$ in $A$ and the set of all subsets of $\operatorname{Spec}(A)$ which are both open and closed in $\operatorname{Spec}(A)$ (cf. [3, Theorem 7.3 and its Corollary]). It follows that $\operatorname{Spec}(A)$ is connected if and only if 0 and 1 are the only idempotents in $A$. In this case $A$ is also called connected.

In the following, let $G$ be a finite group and $R=\bigoplus_{x \in G} R_{x}$ a $G$-graded ring such that $R_{1} \subseteq \mathrm{Z}(R)$. We are going to apply the considerations above with $A=R_{1}$. For $P \in \operatorname{Spec}\left(R_{1}\right)$, we denote by

$$
R_{P}=\left\{\frac{r}{w}: r \in R, w \in R_{1}-P\right\}
$$

the localization of $R$ at $P$. It is easily verified that $R_{P}$ is a $G$-graded ring with $x$-component

$$
\left(R_{P}\right)_{x}=\left(R_{x}\right)_{P}=\left\{\frac{r}{w}: r \in R_{x}, w \in R_{1}-P\right\}
$$

for $x \in G$. The 1-component $\left(R_{P}\right)_{1}=\left(R_{1}\right)_{P}$ of $R_{P}$ is a local ring contained in $\mathrm{Z}\left(R_{P}\right)$, its maximal ideal is

$$
P_{P}=\left\{\frac{p}{w}: p \in P, w \in R_{1}-P\right\},
$$


and the residue field $\left(R_{1}\right)_{P} / P_{P}$ can be identified with the field of fractions of the integral domain $R_{1} / P$ (i.e., with the localization of $R_{1} / P$ at the prime ideal $P / P=0)$. We obtain the following commutative diagram of rings:

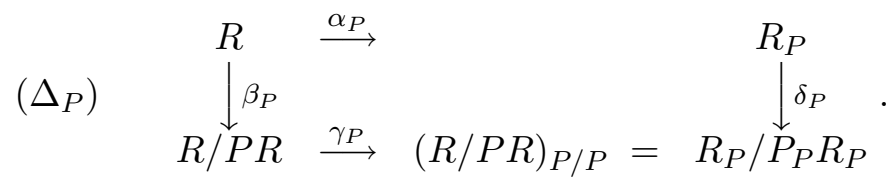

In this diagram all maps are canonical and therefore graded homomorphisms. Both vertical maps are residue class maps, and both horizontal maps can be viewed as canonical maps into localizations.

Lemma 10. Let $P \in \operatorname{Spec}\left(R_{1}\right)$. Then, in the diagram $\left(\Delta_{P}\right)$ above, the kernels of $\gamma_{P}$ and $\delta_{P}$ are contained in the Jacobson radicals of $R / P R$ and $R_{P}$, respectively; in particular, these kernels do not contain any nonzero idempotents.

Proof. The restriction of $\gamma_{P}$ to the 1-components of both $G$-graded rings is just the inclusion map of the integral domain $R_{1} / P$ into its field of fractions. Thus the 1-component of the graded ideal $\operatorname{Ker}\left(\gamma_{P}\right)$ is zero. Hence, by Lemma 6, $\operatorname{Ker}\left(\gamma_{P}\right)$ is nilpotent; in particular, we have $\operatorname{Ker}\left(\gamma_{P}\right) \subseteq \mathrm{J}(R / P R)$.

The kernel of $\delta_{P}$ is $P_{P} R_{P}=\mathrm{J}\left(\left(R_{P}\right)_{1}\right) R_{P}$, and this is contained in $\mathrm{J}\left(R_{P}\right)$ by $[\mathbf{2}$, Corollary $2(\mathrm{c})]$.

We continue to use the notation introduced above.

Lemma 11. Let $P \in \operatorname{Spec}\left(R_{1}\right)$, and let $e$ be an idempotent in $R$. Then $\alpha_{P}(e) \neq 0$ if and only if $\beta_{P}(e) \neq 0$ if and only if $A n n_{R_{1}}(e) \subseteq P$.

Here $\operatorname{Ann}_{R_{1}}(e)=\left\{a \in R_{1}: a e=0\right\}$ denotes the annihilator of $e$ in $R_{1}$, an ideal of $R_{1}$.

Proof. Lemma 10, together with the commutativity of $\left(\Delta_{P}\right)$, implies the following:

$$
\alpha_{P}(e)=0 \Longleftrightarrow \delta_{P}\left(\alpha_{P}(e)\right)=0 \Longleftrightarrow \gamma_{P}\left(\beta_{P}(e)\right)=0 \Longleftrightarrow \beta_{P}(e)=0 .
$$

Moreover, the definition of the localization $R_{P}$ shows:

$$
\alpha_{P}(e)=0 \Longleftrightarrow w e=0 \text { for some } w \in R_{1}-P \Longleftrightarrow \operatorname{Ann}_{R_{1}}(e) \nsubseteq P \text {. }
$$

For an idempotent $e$ in $R$, we set

$$
\begin{aligned}
\mathcal{X}(e) & =\left\{P \in \operatorname{Spec}\left(R_{1}\right): \alpha_{P}(e) \neq 0\right\}=\left\{P \in \operatorname{Spec}\left(R_{1}\right): \beta_{P}(e) \neq 0\right\} \\
& =\left\{P \in \operatorname{Spec}\left(R_{1}\right): \operatorname{Ann}_{R_{1}}(e) \subseteq P\right\}=\mathcal{V}\left(\operatorname{Ann}_{R_{1}}(e)\right) .
\end{aligned}
$$

In case $e \in R_{1}$, we have $\beta_{P}(e) \neq 0$ if and only if $e \notin P$. Thus our notation here is compatible with the notation introduced at the beginning of this section. Moreover, we see that, for any idempotent $e$ in $R, \mathcal{X}(e)$ is closed in $\operatorname{Spec}\left(R_{1}\right)$. Our aim is to show that $\mathcal{X}(e)$ is also open in $\operatorname{Spec}\left(R_{1}\right)$. Our main tool will be the following result. 
Lemma 12. Let e be a nonzero idempotent in $R$, and write $e=\sum_{x \in G} e_{x}$ with $e_{x} \in R_{x}$ for $x \in G$. If $R_{1}$ is a local ring then $e_{x}$ is invertible in $R$ for some $x \in G$.

Proof. Let $M$ denote the maximal ideal of $R_{1}$. Then $R / M R$ is a $G$-graded ring whose 1-component is the field $R_{1} / M$. Since $M R=\mathrm{J}\left(R_{1}\right) R \subseteq \mathrm{J}(R)$ by [2, Corollary 2(c)], we have $e+M R \neq 0$. Moreover, an element $r \in R$ is invertible in $R$ if and only if $r+M R$ is invertible in $R / M R$. Thus we can replace $R$ by $R / M R$ and therefore assume that $R_{1}$ is a field.

Then, by Lemma $9, R\left[G_{R}\right]$ is a crossed product, and $R\left[G-G_{R}\right]$ is a nilpotent graded ideal of $R$. Thus we can also replace $R$ by $R / R\left[G-G_{R}\right]$ and $G$ by $G_{R}$. Therefore we may assume that $R$ is a crossed product. In this situation the assertion is obvious.

The following is the main result of this section.

Proposition 13. If $e$ is an idempotent in $R$ then $\mathcal{X}(e)$ is both open and closed in the Zariski topology of $\operatorname{Spec}\left(R_{1}\right)$.

Proof. We know already that $\mathcal{X}(e)$ is closed, so it suffices to show that $\mathcal{X}(e)$ is open. We write $e=\sum_{x \in G} e_{x}$ with $e_{x} \in R_{x}$ for $x \in G$. If $P \in \mathcal{X}(e)$ then $\alpha_{P}(e)$ is a nonzero idempotent in $R_{P}$. Since the 1-component $\left(R_{1}\right)_{P}$ of $R_{P}$ is a local ring contained in $\mathrm{Z}\left(R_{P}\right)$, Lemma 12 implies that $\alpha_{P}\left(e_{x}\right)$ is invertible in $R_{P}$ for some $x \in G$. Then $a:=e_{x}^{|G|}$ is contained in $R_{1}$, and $\alpha_{P}(a)=\alpha_{P}\left(e_{x}\right)^{|G|}$ is invertible in both $R_{P}$ and $\left(R_{1}\right)_{P}$; in particular, we have $a \notin P$ and $P \in \mathcal{X}(\{a\})$.

Let $Q \in \mathcal{X}(\{a\})$ be arbitrary. Then $a \notin Q$, so $\alpha_{Q}(a)$ is invertible in both $\left(R_{1}\right)_{Q}$ and $R_{Q}$. Since $\alpha_{Q}(a)=\alpha_{Q}\left(e_{x}\right)^{|G|}, \alpha_{Q}\left(e_{x}\right)$ is invertible in $R_{Q}$, too; in particular, we have $\alpha_{Q}(e) \neq 0$, i.e., $Q \in \mathcal{X}(e)$.

This shows that $\mathcal{X}(e)$ contains the open neighborhood $\mathcal{X}(\{a\})$ of $P$. Since $P \in \mathcal{X}(e)$ was arbitrary we conclude that $\mathcal{X}(e)$ is an open subset of $\operatorname{Spec}\left(R_{1}\right)$.

\section{Proofs of the main results.}

We start with a proof of Theorem 2 .

Proof of Theorem 2. We write $\operatorname{Bl}\left(R_{1}\right)=\left\{b_{1}, \ldots, b_{m}\right\}$ and replace each idempotent $e \in E$ by the nonzero elements in $\left\{e b_{1}, \ldots, e b_{m}\right\}$. Then, for $j=$ $1, \ldots, m, R b_{j}=\bigoplus_{x \in G} R_{x} b_{j}$ is a $G$-graded ring such that $R_{1} b_{j} \subseteq \mathrm{Z}(R) b_{j}=$ $\mathrm{Z}\left(R b_{j}\right)$, and $E_{j}:=\left\{e b_{j} \neq 0: e \in E\right\}$ is a set of pairwise orthogonal nonzero idempotents in $R b_{j}$. Moreover, we have $R=\bigoplus_{j=1}^{m} R b_{j}$ and $|E| \leq \sum_{j=1}^{m}\left|E_{j}\right|$. Thus the result will follow if $\left|E_{j}\right| \leq|G|$ for each $j$.

This means that we can replace $R$ by $R b_{j}$ and therefore assume that $R_{1}$ is connected. Then, for $e \in E, \mathcal{X}(e)$ is both open and closed in $\operatorname{Spec}\left(R_{1}\right)$ by Proposition 13. Since $e \neq 0$, we certainly have $\mathcal{X}(e) \neq \emptyset$. (For otherwise Lemma 11 would yield $\operatorname{Ann}_{R_{1}}(e)=R_{1}$ which is impossible.) Since $\operatorname{Spec}\left(R_{1}\right)$ 
is connected this means that $\mathcal{X}(e)=\operatorname{Spec}\left(R_{1}\right)$, so $\beta_{P}(e) \neq 0$ for every $P \in \operatorname{Spec}\left(R_{1}\right)$.

Let $M$ be a maximal ideal of $R_{1}$. Then $\beta_{M}(E)$ is a set of pairwise orthogonal nonzero idempotents in the $G$-graded ring $R / M R$ such that $\left|\beta_{M}(E)\right|=|E|$. Hence we can replace $R$ by $R / M R$ and therefore assume that $R_{1}$ is a field.

In this case, the $G_{R}$-graded ring $R\left[G_{R}\right]$ is a crossed product, and $R\left[G-G_{R}\right]$ is a nilpotent graded ideal of $R$, by Lemma 9 . Thus we can replace $R$ by $R / R\left[G-G_{R}\right]$ and $G$ by $G_{R}$ and therefore assume that $R$ itself is a crossed product. Then $R$ has dimension $|G|$ over the field $R_{1}$. Since $E$ is clearly linearly independent over $R_{1}$ we conclude that $|E| \leq|G|$.

The proof of Theorem 1 is now easy.

Proof of Theorem 1. Let $E$ be a set of pairwise orthogonal nonzero idempotents in $\mathrm{Z}(R)$. It suffices to prove that $|E| \leq|G| \cdot\left|\mathrm{Bl}\left(R_{1}\right)\right|$. The centralizer $C=\mathrm{C}_{R}\left(R_{1}\right)$ of $R_{1}$ in $R$ is a graded subring of $R$ with 1-component $C_{1}=\mathrm{Z}\left(R_{1}\right)$. Moreover, $C$ and $E$ satisfy the hypotheses of Theorem 2 . Thus $|E| \leq|G| \cdot\left|\mathrm{Bl}\left(C_{1}\right)\right|=|G| \cdot\left|\mathrm{Bl}\left(R_{1}\right)\right|$, and we are done.

We note that E.C. Dade has asked (private communication) whether Theorem 1 in this paper and Theorem 2.2 in [1] are just two special cases of a more general result on actions of Hopf algebras.

We now turn to a proof of Theorem 4 .

Proof of Theorem 4. By Proposition 13, $\mathcal{X}(e)$ is a closed and open subset of $\operatorname{Spec}\left(R_{1}\right)$. Thus, by [3, Theorem 7.3], there is a unique idempotent $e_{1}$ in $R_{1}$ such that $\mathcal{X}(e)=\mathcal{X}\left(e_{1}\right)$. Note that

$$
1-e_{1} \in \operatorname{Ann}_{R_{1}}\left(e_{1}\right) \subseteq \bigcap_{P \in \mathcal{X}\left(e_{1}\right)} P=\bigcap_{P \in \mathcal{X}(e)} P=\sqrt{\operatorname{Ann}_{R_{1}}(e)} .
$$

But $\left(1-e_{1}\right)^{2}=1-e_{1}$, so we conclude that $1-e_{1} \in \operatorname{Ann}_{R_{1}}(e)$. Hence we have $\left(1-e_{1}\right) e=0$ and $e=e_{1} e$.

It remains to prove that $e_{1}$ is primitive in $R_{1}$. Thus suppose that $e_{1}=$ $f_{1}+g_{1}$ with orthogonal idempotents $f_{1}, g_{1} \in R_{1}$. Then $e=e_{1} e=f_{1} e+g_{1} e$ with orthogonal idempotents $f_{1} e, g_{1} e$ in $\mathrm{Z}(R)$. Since $e$ is a block idempotent of $R$, we conclude that $f_{1} e=0$ or $g_{1} e=0$. Without loss of generality, we may assume that $g_{1} e=0$. Then $f_{1} e=e$ and $\operatorname{Ann}_{R_{1}}\left(e_{1}\right) \subseteq \operatorname{Ann}_{R_{1}}\left(f_{1}\right) \subseteq$ $\operatorname{Ann}_{R_{1}}(e)$. Thus

$$
\mathcal{V}\left(\operatorname{Ann}_{R_{1}}\left(e_{1}\right)\right) \supseteq \mathcal{V}\left(\operatorname{Ann}_{R_{1}}\left(f_{1}\right)\right) \supseteq \mathcal{V}\left(\operatorname{Ann}_{R_{1}}(e)\right),
$$

i.e., $\mathcal{X}\left(e_{1}\right) \supseteq \mathcal{X}\left(f_{1}\right) \supseteq \mathcal{X}(e)=\mathcal{X}\left(e_{1}\right)$ and therefore $\mathcal{X}\left(e_{1}\right)=\mathcal{X}\left(f_{1}\right)$. So the uniqueness of $e_{1}$ implies that $e_{1}=f_{1}$, and we are done.

Now we combine Theorem 4 with results in [1] in order to prove Theorem 3 . 
Proof of Theorem 3. The centralizer $C=C_{R}\left(R_{1}\right)$ of $R_{1}$ in $R$ is a $G$-graded subring of $R$. Moreover, [1, Theorem 5.8] implies that there is a block idempotent $c$ in $C$ such that $a c=c$. By Theorem 4, there exists a block idempotent $c_{1}$ in $C_{1}=\mathrm{Z}\left(R_{1}\right)$ such that $c=c c_{1}=a c c_{1}$.

But $C$ is also a $G$-ring with $C^{G}=\mathrm{Z}(R)$, and the action of $G$ on $C$ is compatible with the $G$-grading of $C$. Thus the sum of the $G$-orbit of $c_{1}$ is an idempotent $d$ in $C_{1}^{G} \subseteq \mathrm{Z}(R)$ such that $a d c_{1}=a c_{1} \neq 0$. Since $a$ is a block idempotent in $R$ we conclude that $a d=a$. Moreover, $d$ is a sum of finitely many block idempotents of $R_{1}$. Thus $R d$ is a fully $G$-graded ring, and its 1-component $R_{1} d$ has finite block theory. Hence Theorem 1 implies that $R d$ has finite block theory, too.

Since $S$ is a $G$-invariant unitary subring of $R$, it contains $R_{1} 1_{R} R_{1}=R_{1}$. In particular, we have $d \in \mathrm{Z}(S)$. Furthermore, $S d$ is a $G$-invariant unitary subring of $R d$, and $a$ is a block idempotent in $R d$. Now [1, Proposition 9.2] implies that $S d$ has finite block theory. We write $d=b_{1}+\cdots+b_{n}$ with block idempotents $b_{1}, \ldots, b_{n}$ of $S d$ (and $S$ ). Then $a b_{i} \neq 0$ for some $i \in\{1, \ldots, n\}$, and we have found a block idempotent $b=b_{i}$ of $S$ such that $a b \neq 0$.

By $[\mathbf{1}$, Proposition 8.3], $\mathrm{Z}(S)$ is a unitary $G$-subring of $C$. Thus, for $x \in G$, ${ }^{x} b$ is a block idempotent in $S$ such that $a\left({ }^{x} b\right)={ }^{x}(a b) \neq 0$. Moreover, the sum of the $G$-orbit $B$ of $b$ is an idempotent $e$ in $\mathrm{Z}(S)^{G} \subseteq C^{G}=\mathrm{Z}(R)$ such that $a e \neq 0$. Since $a$ is a block idempotent in $R$, we conclude that $a e=a$. Hence every block idempotent $b^{\prime}$ of $S$ such that $a b^{\prime} \neq 0$ satisfies $e b^{\prime} \neq 0$ and is therefore contained in $B$. We are done.

It remains to prove Theorem 5 .

Proof of Theorem 5. (i) This is a consequence of Theorem 3, applied with $S=R_{1}$.

(ii) Let $e_{1}$ be a block idempotent of $R_{1}$, and note that $G$ acts on $C_{1}=$ $\mathrm{Z}\left(R_{1}\right)$. Thus the $G$-orbit $B$ of $e_{1}$ is finite, and the sum of the elements in $B$ is an idempotent $d$ in $C_{1}^{G} \subseteq \mathrm{Z}(R)$. Moreover, $R d=\bigoplus_{x \in G} R_{x} d$ is a fully $G$-graded ring whose 1-component $R_{1} d$ has finite block theory. Hence $R d$ has finite block theory as well by Theorem 1 .

(iii) It is easy to see that a block idempotent $e$ of $R$ satisfies $e e_{1} \neq 0$ if and only if $e \in R d$. Thus this part is a consequence of [1, Theorem 8.10], applied to the $G$-invariant subring $R_{1} d$ of the fully $G$-graded ring $R d$.

(iv) This is a consequence of $[\mathbf{1}$, Theorem 8.12].

Acknowledgements. Most of this work was done while the first author was visiting the Universität Jena, April-July 1998, supported by the DAAD. He thanks the DAAD very much for the financial support. He is also grateful to the Mathematisches Institut, Universität Jena, and to the second author for their hospitality. Both authors would like to thank E.C. Dade for several useful comments concerning an earlier version of this paper, and the referee for a careful reading of this manuscript. 


\section{References}

[1] E.C. Dade, Blocks of fully graded rings, Pacific J. Math., Olga Taussky-Todd memorial issue, (1997), 85-122.

[2] P. Grzeszczuk, On G-systems and G-graded rings, Proc. Amer. Math. Soc., 95 (1985), $248-252$.

[3] N. Jacobson, Basic Algebra II, Second Edition, W.H. Freeman Company, New York, 1989.

[4] E.R. Puczylowski, A note on graded algebras, Proc. Amer. Math. Soc., 113 (1991), $1-3$.

Received January 28, 1999 and revised April 8, 1999.

Department of Mathematics

WUHAN UNIVERSITY

WUHAN 430072

P. R. CHINA

E-mail address: yunfan@whu.edu.cn

Mathematisches Institut

UNIVERSITÄT JENA

07740 JENA

GERMANY

E-mail address: kuelsham@maxp04.mathe.uni-jena.de 